\title{
Indications and techniques for anterior cervical plating
}

\author{
John M. Rhee, Jong-Beom Park', Jun-Young Yang ${ }^{2}$, K. Daniel Riew ${ }^{3}$
}

Department of Orthopaedic Surgery, Emory Spine Center, Emory University School of Medicine, Atlanta, GA, USA, 'ĳongbu St. Mary's Hospital, The Catholic University School of Medicine, ${ }^{2}$ Chungnam National University Hospital, Daejeon, Korea, and ${ }^{3}$ Washington

University School of Medicine, St. Louis, MO, USA

Anterior cervical plating is commonly performed to stabilize anterior cervical fusions. Modern plating options include dynamic plates, with screws that can either toggle within fixed holes or translate within slotted holes. Regardless of the plating system used, paramount to success and avoidance of complications with plated anterior cervical fusions are meticulous plating techniques, exacting graft carpentry, and understanding the biomechanical limitations of plating in certain situations, such as multilevel corpectomies reconstructed with a single-strut graft. In order to prevent graft-related complications associated with long-strut grafts, additional posterior fixation and fusion, or alternative corpectomy constructs, such as multilevel anterior cervical discectomy and fusion, corpectomydiscectomy, and corpectomy-corpectomy, should be considered instead if the pattern of stenosis allows.

Key words: Anterior cervical fusion; cervical plate; surgical technique; surgical indication

Anterior cervical plating has numerous potential benefits as an adjunct to anterior cervical discectomy and fusion (ACDF). It provides rigid fixation, resists graft settling and development of segmental kyphosis, promotes higher fusion rates, allows for less cumbersome external immobilization, and reduces the incidence of graft extrusion. However, anterior plating also has potential downsides: it adds to surgical time and cost, and makes revision anterior surgery more difficult if the plate must be removed (Figure 1). In addition, there are a number of potential implant-related complications that can occur with plating, such as esophageal erosion from loose plates and screws, adjacent level or peri-plate ossification (Figure 2) from plates that abut adjacent disc spaces, and errant screws that may injure adjacent discs, nerve roots, spinal cord, or vertebral artery.

Although anterior cervical plating seems to be a straightforward procedure, the potential for complications is high if proper attention

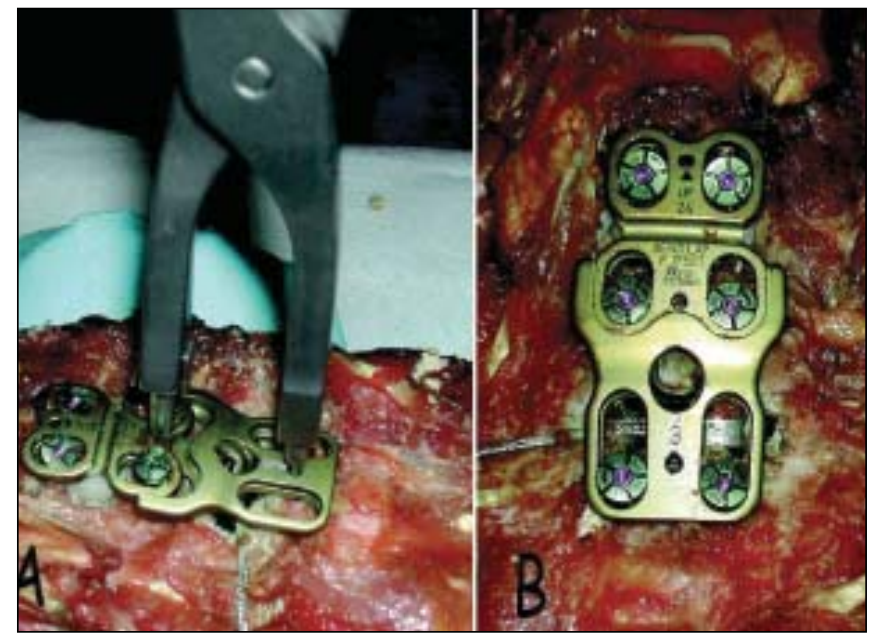

Figure 1: Traditionally, if adjacent segment surgery is needed, the old plate must be removed and replaced with a longer plate. The E-plate (Aesculap, Tuttlingen, Germany) allows one to extend the fusion to another level without having to remove the original plate. Figure courtesy of Ron Apfelbaum

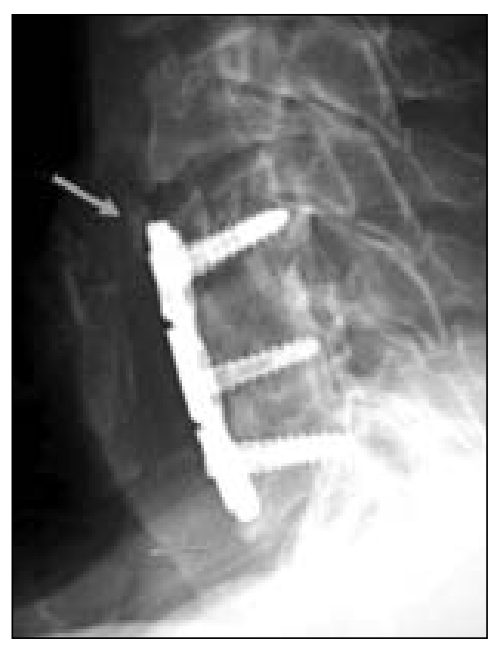

Figure 2: Peri-plate ossification can occur if the plate is too close $(<5 \mathrm{~mm})$ to adjacent disc spaces. Particular attention to avoiding this complication must be paid when using subsidence plates like this one by placing the plate far away enough from adjacent discs to allow for settling without overlap 
to detail is not paid during surgical planning and intraoperatively. Plating should not necessarily be relied upon to compensate for poor graft carpentry, nor can it reliably produce clinical success in the face of biomechanically unsound constructs or those in which appropriate consideration has not been given to biologic factors such as graft type vis-a-vis host healing potential. In this article, we review considerations in performing anterior cervical plating, and discuss technical and biomechanical factors associated with successful use.

\section{Indications for anterior cervical plating}

Despite widespread use of anterior cervical plates since their introduction by Bohler, ${ }^{[1]}$ there is no true consensus as to its indications. Plates are commonly used in the treatment of degenerative disorders of the cervical spine, tumors, trauma, and deformity. Although the rationale for anterior plate fixation in reconstructing unstable conditions such as tumors or fractures seems obvious, it remains unclear whether anterior plating is truly of benefit in many common spondylotic conditions of the cervical spine. Several studies have failed to demonstrate any difference in union rates for single-level plated ACDF using autograft, ${ }^{[2-4]}$ although plating may allow for less graft collapse and segmental kyphosis as the graft heals. ${ }^{[2]}$ In contrast, the literature does in general suggest higher fusion rates with plating of multilevel ACDFs (i.e., two or more segments). ${ }^{[5,6]}$ The literature also suggests benefit to plating ACDFs at one or more levels performed with allograft only. $^{[7]}$

Anterior plates are also commonly used to stabilize corpectomy constructs in addition to ACDFs. A one- or two-level plated corpectomy generally leads to acceptable outcomes. However, three or more level plated corpectomies have been reported to have extremely high failure rates. Although it may seem logical to suspect that the addition of an anterior cervical plate might reduce the incidence of graft-related complications in multilevel strut graft reconstructions, clinical series of plated multilevel corpectomies ${ }^{[8]}$ have actually been associated with higher graft complication rates than the unplated counterparts. ${ }^{[9]}$ In one study, graft displacement rates were $9 \%$ for a two-level corpectomy vs $50 \%$ in three-level corpectomies despite anterior plating. ${ }^{[8]}$ Biomechanical studies which shed light on these clinical failures suggest that long-plated strut graft constructs may be mechanically unfavorable because they rapidly lose stability under fatigue loading. ${ }^{[10]}$ Multilevel plated corpectomies have also been demonstrated in vitro to reverse load transfer through the strut graft, such that the graft is paradoxically unloaded in flexion and loaded to supraphysiologic levels in extension. ${ }^{[11]}$ Taken together, these studies suggest that plating cannot compensate for an insurmountable biomechanical milieu. In patients with multilevel stenosis, instead of performing a multilevel plated corpectomy with a single, long-strut graft, consideration should be given to posterior approaches such as laminoplasty, combined anterior decompression with posterior fixation, or, if anterioronly surgery is preferred, the use of alternative corpectomy constructs as described below.

\section{Alternative corpectomy constructs}

If an anterior-only approach is chosen for the patient with stenosis arising from multiple segments, cervical plating can still be performed. However, due to the high failure rates of plated multilevel corpectomies using a single, long-strut graft, consideration should be given to alternative corpectomy constructs which have been designed to reduce the incidence of graft and plate complications. Multilevel ACDF is one alternative that can be performed if the stenosis is disc-based and resection of the posterior vertebral body is not necessary. Advantages over a singlelong strut include the ability to achieve better fixation with segmental screw placement into every vertebral body within the construct, and better preservation or even recreation of lordosis. ACDF grafts, in comparison to long struts, are also much less likely to dislodge. The major disadvantage may be a higher pseudarthrosis rate due to the increased number of bony surfaces requiring healing (e.g., six bony surfaces for a three-level ADCF from $\mathrm{C} 4$ to $\mathrm{C} 7 \mathrm{vs}$ two surfaces for a two-level corpectomy with single-strut graft from $\mathrm{C} 4$ to $\mathrm{C} 7$ ), but the literature is not uniform on this point. ${ }^{[7,12]}$

Another alternative when dealing with compression arising from three disc levels is to perform a single-level corpectomy at two disc levels, then an ACDF at the remaining level (i.e., corpectomydiscectomy). ${ }^{[13]}$ The corpectomy-discectomy construct is a compromise solution which avoids the biomechanical issues of a single-long strut and at the same time decreases the number of healing surfaces by two versus a multilevel ACDF. Segmental plate and screw fixation is achievable at every level except for the corpectomy level. If the patient's compressive pathology does not dictate otherwise, the corpectomy is performed at the upper two levels in order to avoid the mechanical disadvantage of having a corpectomy at the bottom end of the construct, where it would be more likely to dislodge.

A final alternative for pathology involving four disc levels is a double corpectomy (i.e., two single-level corpectomies separated by an intact intervening vertebra (corpectomy-corpectomy). ${ }^{[12]}$ This construct achieves fewer healing surfaces versus multilevel ACDF, and at the same time avoids a single-long strut. Fixation is obtained in three vertebrae: at the top, bottom, and middle of the construct. Before contemplating the use of one of these alternative corpectomy constructs, however, the surgeon must ensure that the pattern and location of the patient's stenosis are appropriate to the procedure.

\section{Plating options}

Rigid plates with fixed angle screws were historically the first ones used in the cervical spine (Figure 3). A plate beneficially provides a block to extension and prevents excessive settling, but a rigidly fixed plate can also undesirably prevent compression and loading of a graft during the natural course of healing. In order to better accommodate settling, buttress plating has been recommended by some authors as an alternative means of stabilizing long-anterior strut grafts. ${ }^{[14]}$ The rationale for their use is that, whereas a spanning plate fixed to the vertebral body above and below the graft can prevent graft settling as the graft 
heals and thus act as a 'distraction device,' a buttress plate is fixed only at one end of the construct and thus can allow for settling to occur while preventing graft extrusion. Although theoretically appealing, buttress plates as stand alone anterior fixation devices have been associated with catastrophic complications. ${ }^{[15]}$ When the caudal part of the graft extrudes, it can force the cranial part of the plate to extrude into the pharynx, causing tracheal compression, and potential airway compromise (Figure 4). For this reason, we strongly recommend against their use in anterioronly cases.

Dynamic plates are another alternative to rigid plate fixation. They also allow for settling to occur and are again theoretically appealing, but strong clinical evidence regarding efficacy in stabilizing long-strut grafts is currently lacking. Depending on plate design and configuration, dynamic plates may allow for bidirectional or unidirectional subsidence. Bidirectional subsidence can occur if the plate has slotted holes at the most proximal and distal ends (e.g., ABC plate, Aesculap, Tuttlingen, Germany) (Figure 5), or if the proximal and distal ends of the plate can

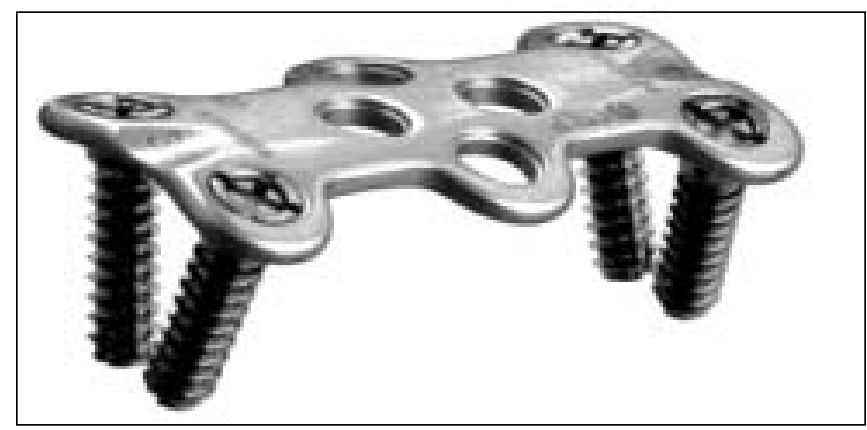

Figure 3: The original CSLP plate (Synthes, Paoli, PA) used fixed angle screws only. The screws are locked to the plate by the secondary inner set screws

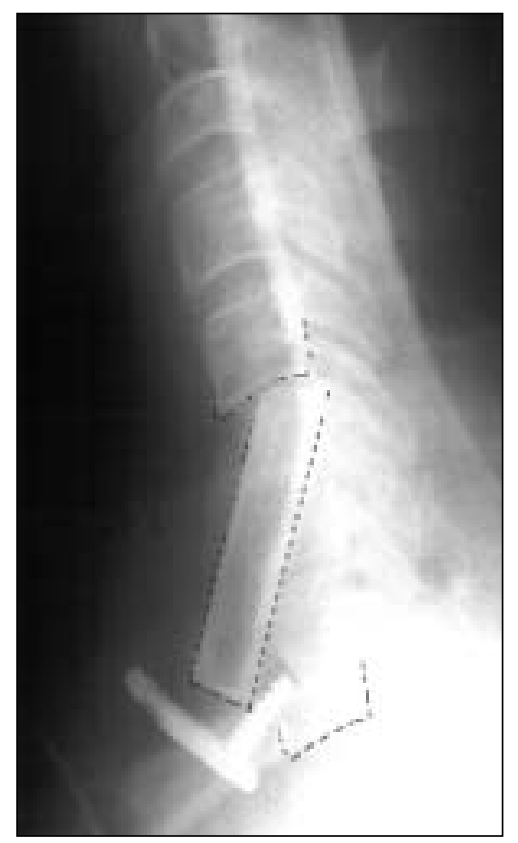

Figure 4: Catastrophic failure of a buttress plate used to stabilize cervical corpectomy telescope with respect to each other (e.g., DOC plate, Depuy Acromed, Raynham, MA) (Figure 6). Unidirectional subsidence occurs in plates that have a slotted hole at one end only (e.g., CTek, Interpore Cross, Irvine, CA) (Figure 7). Concerns associated with the use of dynamic plates include excessive settling leading to plates overlapping and injuring adjacent disc spaces, as well as kyphosis, foraminal stenosis, and construct failure. Yet another alternative is to use variable angle screws in a nonslotted plate. These screws toggle within the plate as the graft settles, allowing for some degree of subsidence, but, unlike in slotted or translational systems, the screws do not translate longitudinally within the plate. This option is attractive for those who are concerned about uncontrolled and excessive settling but desire greater load sharing than that offered by the traditional rigid plate with fixed angle screws.

\section{Technical points \\ Graft carpentry: ACDF}

As mentioned, rigid plates can prevent settling as a graft heals,

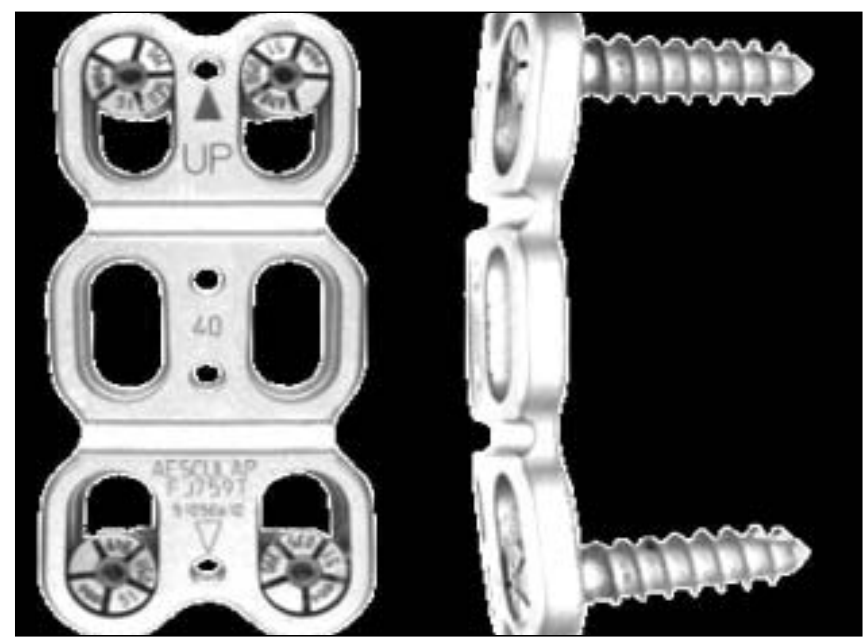

Figure 5: $A B C$ plate. Slotted holes in this plate allow for bidirectional settling of the construct and subsidence of graft

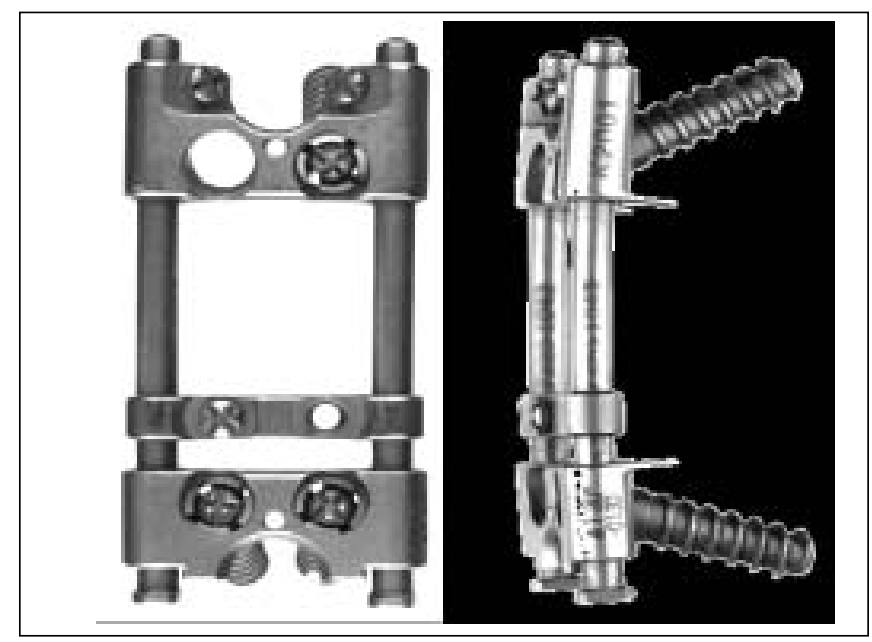

Figure 6: DOC plate. The proximal and distal ends of this plate slide along the rods, allowing for bidirectional subsidence 
thereby acting as a distraction device and paradoxically preventing union. Subsequently, use of a rigid plate may require even better graft and endplate carpentry than the unplated counterpart. The primacy of proper graft technique in achieving success with plated anterior cervical fusions is paramount. In order to achieve intimate contact of bone graft with the endplates, a rectangular space is created with parallel decorticated endplates (Figure 8). A highspeed burr is helpful in fashioning this construct. Because the inferior endplate tends to be concave, removal of the anterior inferior lip is often necessary in order to obtain an unobstructed view into the back of the disc space for decompression and also to allow the passage of a graft appropriately sized to match the larger height present at the center of the disc space. The endplate is decorticated to bleeding bone back to the posterior longitudinal ligament. The central 1-2 mm of the posterior lip can be removed if necessary for cord decompression but does not routinely need to be removed and can act as a buttress against the unlikely occurrence of posterior graft displacement. The preoperative

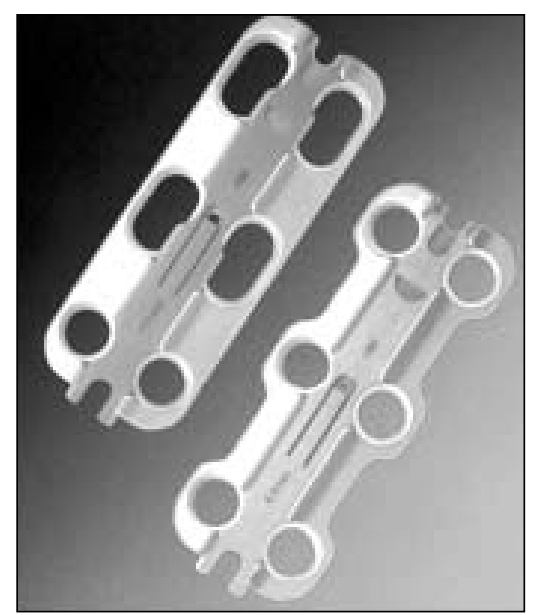

Figure 7: C-Tek plate. Two versions are shown. One (left) allows for unidirectional subsidence through the slotted holes, and the other (right) has fixed screw holes. Variable screws that toggle within the fixed holes do not translate longitudinally but still allow for subsidence, albeit to a lesser degree than that which occurs using slotted holes

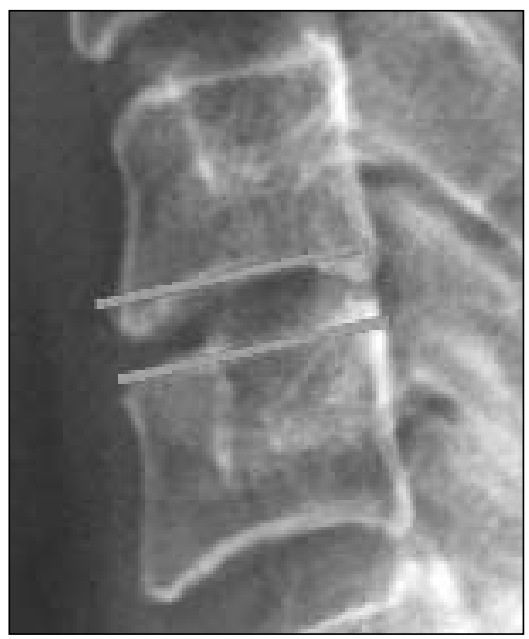

Figure 8: Optimal preparation of endplates to achieve intimate grafthost contact lateral radiograph can be used to estimate the amount of anterior inferior endplate resection. It is important not to remove too much bone off of the inferior endplate, however, as doing so limits the bone available in the vertebra to accommodate a plate and screws without violating the proximal uninvolved disc space. In contrast to the inferior endplate, because the superior endplate tends to be less concave, aggressive bone resection in this area is not necessary. Thorough decortication of both endplates is desirable to enhance the chance of successful fusion. We prefer to use a microscope, and tilt the scope as necessary to get clear views of each endplate during this procedure. Irrigation is performed to help remove bone debris with suction as well as prevent thermal necrosis to bone and heat transmission to the neural elements.

It is important to remember that the key step in most anterior cervical procedures is an adequate neural decompression. A thorough discectomy should be performed to relieve the compressive pathology. If a soft, extruded disc herniation is present, we recommend retracting the posterior longitudinal ligament, visualizing the root and cord, and verifying that all fragments have been removed. If the problem is uncinate osteophytosis and foraminal stenosis, resection of the PLL is not routinely necessary but can be performed if any question remains as to the adequacy of decompression. In patients with ossification of the posterior longitudinal ligament (OPLL), the PLL should either be resected or allowed to float ${ }^{[16]}$ anteriorly if resection would lead to a large dural deficit. Biomechanical studies have demonstrated that the PLL does provide some ligamentous stability to flexion. ${ }^{[17]}$

\section{Sizing and placing bone graft}

One way to theoretically enhance fusion rates is to place as much bone in the interspace as possible. Some of the commercially available dense cancellous and corticocancellous grafts are fairly wide and help in achieving this goal. However, if fibular allograft is used, our preference is to place two structural grafts side by side into each disc space whenever possible, because the width of a single-fibular graft is typically small compared to the width of the disc space. A fairly wide dissection of the longus colli and discectomy is necessary, with greater resection of the uncinates, to create the desired width for two grafts. A penfield dissector can be used to identify the lateral edge of the uncinate during dissection to avoid straying beyond it and causing vertebral artery laceration. It is a good idea to preoperatively scrutinize the position of the vertebral arteries on CT scan or MRI to be certain that a medial aberrancy does not exist. Typically, medial anomalies arise from tortuosities within the substance of the vertebral body and come into play during corpectomy rather than discectomy. ${ }^{[18]}$ Based upon preoperative estimates of the desired graft heights, a range of graft heights are cut while the patient is being prepped and draped or during exposure to minimize surgical time. Estimates are made from the preoperative lateral radiograph: in most cases, a height of 1-2 mm greater than that measured will suffice, and the depth of the graft should be about $2 \mathrm{~mm}$ less than $90 \%$ of the measured anterior-posterior diameter of the vertebral body (which 
takes into account a radiographic magnification rate of $10 \%$ ). Usual graft heights range from 7 to $10 \mathrm{~mm}$. Care is taken to ensure that the heights are evenly cut to maximize endplate contact and prevent tilting of the disc space and subsequent scoliosis from one graft being taller than the other.

The final height of the graft can be determined after decortication with graft sizers that manufacturers provide for use with commercially prepared allografts. Vertebral body pin distraction is helpful. Overdistraction of the disc space is not desirable and can be associated with severe postoperative neck pain, presumably from posterior capsular distraction. A few clicks on the distraction device will suffice. The sizer is then gently impacted with light strokes on a mallet. A snug fit in the distracted position will assure an excellent fit after removal of distraction. If a sizer does not fit into the disc space but the next size smaller is too loose, the surgeon should determine the area of impingement within the disc space and consider burring gently in that region to allow safe passage of the graft. The uncinates are typically the areas where the graft may not fit, due to the surgeon's natural and prudent desire to avoid vertebral artery injury. The surgeon should keep in mind that the goal in this situation is to fine tune the placement of graft, not to make a tall graft fit into a disc space which is grossly mismatched. Particularly if a Robinson type autograft ${ }^{[19]}$ or cancellous allograft is used, the height should be at least $6 \mathrm{~mm}$ to preserve proper mechanical properties and prevent graft collapse with loading. This height will also help enlarge foraminal height. The graft is never forced into position in order to avoid neurologic injury and potential graft fracture during insertion.

For multilevel ACDF, it is easiest to begin at the most caudal level and place the graft immediately after decompression prior to working on the next proximal interspace. As the most caudal level of a multilevel ACDF is most likely to go on to nonunion, placing graft there first will ensure an even better fit due to the compression achieved as the subsequent graft is impacted into the interspace above.

\section{Fusion techniques for corpectomy}

The width of the corpectomy is determined by that of the spinal cord requiring decompression. At the level of the disc spaces, a relatively wide decompression, which relieves both cord and foraminal stenosis is recommended. However, the width of the corpectomy within the vertebral body need not be that wide, as doing so invites potential vertebral artery injury and also unnecessarily diminishes the potential for side to side healing of the graft to the vertebral bodies.

The endplates should be thoroughly decorticated as described for ACDF to promote the optimal environment for fusion. They can be sculpted in a parallel fashion, or with central cutouts (Figure 9) to lock the graft into place and prevent dislodgement. With the cutout technique, a 3-4 mm deep slot is fashioned into the caudal and cephalad endplates with a burr. If desired, the anterior portion of the inferior body can be notched to admit the caudal end of the graft with less distraction than would be necessary in the absence of the notch. Particularly if the caudal end of the construct arises at a relatively lordotic vertebral body (e.g., C7), the cutout technique is better suited to resist graft kickout. Regardless of which levels are operated upon, the caudal end of the graft should ideally sit parallel to the floor in order to avoid shear forces promoting dislodgement. This can be done by making the slot within the vertebral body parallel to the floor, even if the vertebral body is not.

A graft is measured which will perfectly span the corpectomy. The graft should be wide enough to fill approximately two-thirds of the width of the vertebral body, as this width is sufficient to cover the space created by decompressing the cord. In the anteriorposterior plane, if a subtotal corpectomy is performed, the diameter of the graft should be approximately $5 \mathrm{~mm}$ smaller than the depth of the vertebrae. The posterior $2-3 \mathrm{~mm}$ of the vertebral body can then be left intact and the graft placed flush against this cortex, still allowing the graft to be recessed about 2-3 $\mathrm{mm}$. If a total corpectomy is performed, care is taken to find a graft, which will fill most of the depth of the vertebral body but still be small enough to stay well clear of the decompressed cord when recessed by $2-3 \mathrm{~mm}$ from the front of the vertebral body.

Various devices can be used to measure the length of the corpectomy graft. The wooden end of a cotton applicator can be whittled away until it just fits into the corpectomy and used as a template for cutting the graft. Gardner-Wells tong traction can be helpful in stabilizing the head and neck when performing corpectomy. Five pounds of weight is used to steady the head initially, and then weight is added after decompression to allow for proper graft insertion. Typically, 30-40 pounds of traction will suffice. Significant traction should be avoided until the cord is decompressed in order to avoid the potential for cord compression. The graft is inserted into the cephalad vertebral body, and then gently tamped into position under distraction into the caudal vertebral body. An alternative to traction is the use of vertebral body distraction pins. Care must be taken when using these pins in the face of osteoporotic bone, as the pins can plow through the

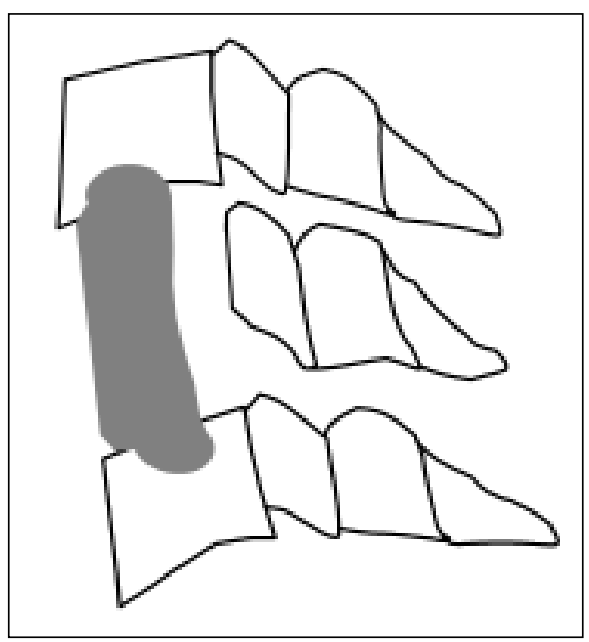

Figure 9: Notches or cutouts can be created into the endplates with matching surfaces on the graft when reconstructing corpectomies in order to achieve a more stable construct 
vertebrae after application of distraction. In addition, especially at the caudal and cephalad vertebrae, the pin hole should be placed centrally to avoid the more lateral plate fixation sites so that good screw purchase can still be achieved after removal of the pins.

The vertebral body bone resected during corpectomy can be used to fill the spaces in and around the structural member: Because bony union is desirable not only at the ends of the construct but also side to side between the shaft of the strut graft and the remaining vertebral bodies, intimate fit of graft to host is desirable in all regions. The central marrow cavity of the structural graft is filled with local autograft and/or demineralized bone matrix. Cages can also be used in lieu of structural bone grafts. ${ }^{[20]}$ The cages can be filled with local autograft from corpectomy, or a combination of autograft, allograft, and bone graft substitutes such as demineralized bone matrix preparations. The principles of proper carpentry, however, remain regardless of the specific interbody device used. If autograft is scarce, it is best to save it for the ends of the strut and fill the central portion of the marrow cavity with a bone graft substitute. The uncinate regions at each disc level are a good surface for fusion and can be grafted with local bone. Care must be taken, however, if the posterior longitudinal ligament has been resected and persistent epidural bleeding exists, as a closed space may form if the uncinates are tightly packed and lead to the potential for epidural hematoma.

\section{Plating techniques}

Once the graft has been placed, the size of the plate is determined. Proper plate sizing has several implications. If the plate is too short, the screw may enter the operative disc space and potentially fracture

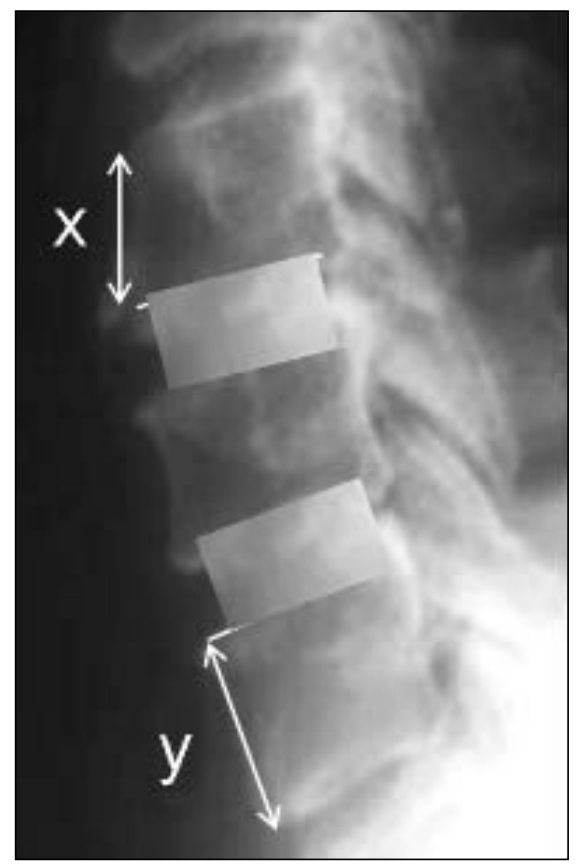

Figure 10: The cranial vertebra is typically shorter than the caudal vertebra. In addition, proper endplate preparation further shortens the cranial $(x)$ vs the caudal vertebra $(y)$. This leaves less room for plates and screws on the cranial vertebra without overlapping the suprajacent uninvolved disc space or displace the graft. If the plate is too long, screws may enter adjacent disc spaces or the plate may overlap the adjacent disc.

Ideally, a plate should be chosen of such length so that the most cephalad and caudad screw holes are immediately adjacent to their respective endplates. Doing so will allow the use of longer screws which angle away from the endplate, and also prevent the plate from overlapping adjacent disc spaces. If a dynamic plate is used, it is even more important to place these screw holes adjacent to the endplate to allow for plate migration with settling of the entire construct. Because proper graft carpentry typically requires greater preparation of the inferior endplate of the cephalad vertebra, the amount of space remaining for a plate and screws will be less on the cephalad $v$ s caudal vertebra (Figure 10). Thus when using a dynamic plate, we prefer to place the slotted portion on the inferior vertebral body to allow for settling of the plate without overlapping adjacent disc spaces (Figure 11). The screw holes in the most cephalad vertebral body are either fixed or variable but not slotted, and those in the intervening segments are either slotted or variable.

The plate should be appropriately contoured into lordosis so that it will lie flush against the vertebral bodies and prevent prominence and esophageal injury. It should be centered coronally within the margins of the uncinate processes. Careful exposure of the uncinates will help in centering the plate. Palpation of the sternal notch can also assist, as can palpating the patient's nose if he has been positioned without rotating the head. The screws at each level should be angled medially in order to avoid nerve root or vertebral artery injury laterally. If the plate is placed too far off center, even properly angled screws of appropriate length may

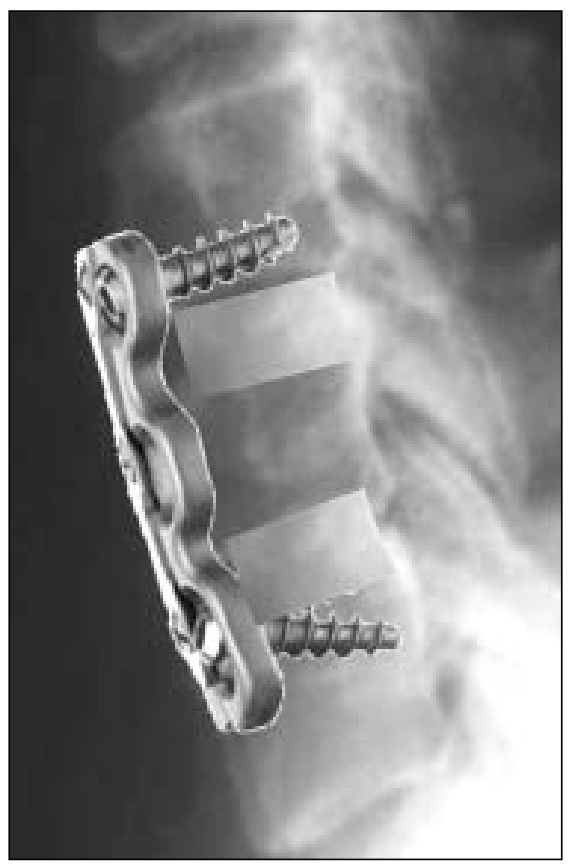

Figure 11: Due to the constraints of vertebral body size and graft carpentry noted in Figure 10, we prefer to place the slotted hole on the inferior end of the construct rather than in the cephalad vertebra, because there is greater room for translation caudally without adjacent disc space overlap due to the larger size of the caudal vertebra 
inadvertently enter the neural or transverse foraminae. The length of the screws can be determined preoperatively from measurements off of CT scan, MRI, or plain radiographs. We prefer to estimate screw length by first measuring the depth of the vertebral body on a preoperative lateral radiograph and subtracting approximately $10 \%$ for magnification. Then, two needles bent to that measurement minus $2 \mathrm{~mm}$ (to provide an extra margin of safety) are placed into two disc spaces for the localizing radiograph intraoperatively. The length of the screw is then determined by comparing the known length of the needles with that of the vertebral bodies on this radiograph. Alternatively, one can make estimates from intraoperative X-rays taken with Caspar pins of known length placed into the vertebral bodies. Either technique provides for maximal unicortical screw length without the risks of bicortical placement, which, with modern locking plates, is not generally necessary (Figure 12). Bicortical screws may be considered in highly unstable situations, trauma, or to achieve adequate purchase in osteopenic bone. Tapping the screw can but does not necessarily need to be performed with most systems. The plate is then locked, and an intraoperative radiograph is taken to ensure proper placement.

\section{Complications of plating}

Catastrophic complications related to anterior cervical plating are thankfully rare but include dislodgement of buttress plates leading to severe airway obstruction and death ${ }^{[15]}$ and esophageal erosion from bone graft or plate irritation. ${ }^{[21]}$ If serial radiographs demonstrate progressive backout of screws, they should be removed in order to prevent esophageal injury. Adjacent level or peri-plate ossification has been reported if the plate is too close to the adjacent disc ( $5 \mathrm{~mm}$ or less $).{ }^{[22]}$ Particularly if a dynamic or subsidence plate is used, the importance of proper plate sizing becomes paramount in order to avoid this complication. Injury to adjacent disc spaces from errant screws is generally preventable with careful attention to technique and usually occurs from using plates that are too long.

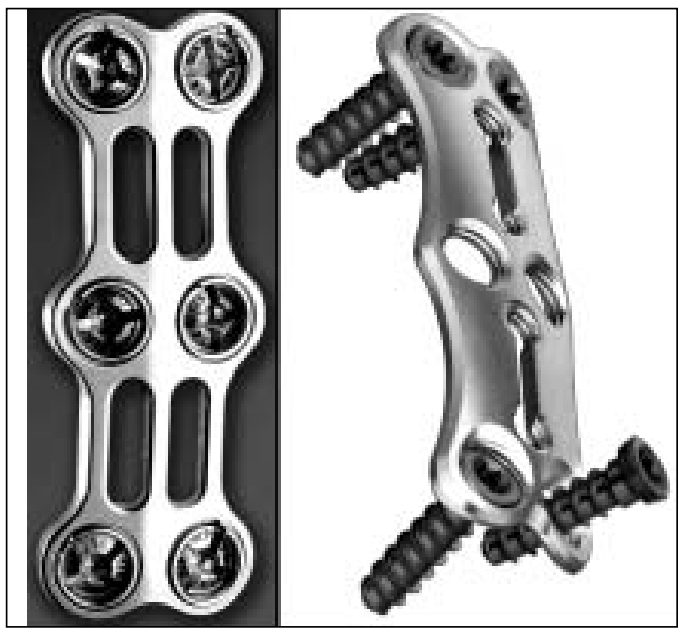

Figure 12: Modern locking plates such as the Vuelock (left; EBI, Parsippany, NJ) and ACLP (right; Synthes, Paoli, PA) utilize one-step locking mechanisms. The screws lock directly to the plate without secondary locking screws or other mechanisms

\section{Conclusion}

Anterior cervical plating can be a useful adjunct to anterior cervical fusions. Meticulous plating techniques are necessary to ensure optimal use. However, perhaps even more important to success and avoidance of complications are exacting graft carpentry and understanding of the biomechanical limitations of plating in certain situations, such as multilevel corpectomies reconstructed with a single-strut graft.

\section{References}

1. Bohler J, Gaudernak T. Anterior plate stabilization for fracture-dislocations of the lower cervical spine. J Trauma 1980;20:203-5.

2. Wang .JC, McDonough PW, Endow K, Kanim LE, Delamarter RB. The effect of cervical plating on single-level anterior cervical discectomy and fusion. J Spinal Disord 1999;12:467-71.

3. Samartzis D, Shen FH, Lyon C, Phillips M, Goldberg EJ, An HS. Does rigid instrumentation increase the fusion rate in one-level anterior cervical discectomy and fusion? Spine .J 2004;4:636-43.

4. Grob D, Peyer JV, Dvorak J. The use of plate fixation in anterior surgery of the degenerative cervical spine: a comparative prospective clinical study. Eur Spine J 2001;10:408-13.

5. Wang JC, MeDonough PW, Endow KK, Delamarter RB. Increased fusion rates with cervical plating for two-level anterior cervical discectomy and fusion. Spine $2000 ; 25: 41-5$

6. Wang JC, MeDonough PW, Kanim LE, Endow KK, Delamarter RB. Increased fusion rates with cervical plating for three-level anterior cervical discectomy and fusion. Spine 2001;26:643-7.

7. Samartzis D, Shen FH, Matthews DK, Yoon ST, Goldberg E.J, An HS. Comparison of allograft to autograft in multilevel anterior cervical discectomy and fusion with rigid plate fixation. Spine J 2003;3:451-9.

8. Vacearo AR, Falatyn SP, Scuderi G.J, Eismont FJ, McGuire RA, Singh K, Garfin SR. Early failure of long segment anterior cervical plate fixation. J Spinal Disord 1998;11:410-5.

9. Emery SE, Bohlman HH, Bolesta MJ, Jones PK. Anterior cervical decompression and arthrodesis for the treatment of cervical spondylotic myelopathy. Two to seventeen-year follow-up. J Bone Joint Surg Am 1998;80:941-51.

10. Isomi T, Panjabi MM, Wang JL, Vacearo AR, Garfin SR, Patel T. Stabilizing potential of anterior cervical plates in multilevel corpectomies. Spine 1999;24:2219-23.

11. DiAngelo DJ, Foley KT, Vossel KA, Rampersaud YR, Jansen TH. Anterior cervical plating reverses load transfer through multilevel strut-grafts. Spine 2000;25:78395.

12. Hilibrand AS, Fye MA, Emery SE, Palumbo MA, Bohlman HH. Increased rate of arthrodesis with strut grafting after multilevel anterior cervical decompression. Spine $2002 ; 27: 146-51$.

13. Riew K. Microscope-assisted anterior cervical decompression and plating techniques for multilevel cervical spondylosis. Operative Techniques in Orthopaedies 1998;8:22-33.

14. Vanichkachorn JS, Vacearo AR, Silveri CP, Albert T.J. Anterior junctional plate in the cervical spine. Spine 1998;23:2462-7.

15. Riew KD, Sethi NS, Devney J, Goette K, Choi K. Complications of buttress plate stabilization of cervical corpectomy. Spine 1999;24:2404-10.

16. Kamikozuru M. [Significance of the anterior floating method for cervical myelopathy due to the ossification of the posterior longitudinal ligament]. Nippon Seikeigeka Gakkai Zasshi 1991;65:431-40.

17. McAfee PC, Cunningham B, Dmitriev A, Hu N, Woo Kim S, Cappuccino A, et al. Cervical dise replacement-porous coated motion prosthesis: a comparative biomechanical analysis showing the key role of the posterior longitudinal ligament. Spine 2003;28):S176-85.

18. Smith MD, Emery SE, Dudley A, Murray K.J, Leventhal M. Vertebral artery injury during anterior decompression of the cervical spine. A retrospective review of ten patients. J Bone Joint Surg Br 1993;75:410-5.

19. Smith GW, Robinson RA. The treatment of certain cervical-spine disorders by anterior removal of the intervertebral disc and interbody fusion. J Bone Joint Surg Am 1958;40:607-24.

20. Riew KD, Rhee JM. The use of titanium mesh cages in the cervical spine. Clin Orthop Relat Res 2002;394:47-54.

21. Smith MD, Bolesta M.J. Esophageal perforation after anterior cervical plate fixation: a report of two cases. J Spinal Disord 1992;5:357-62.

22. Park JB, Cho YS, Riew KD. Development of adjacent-level ossification in patients with an anterior cervical plate. J Bone Joint Surg Am 2005;87:558-63. 